
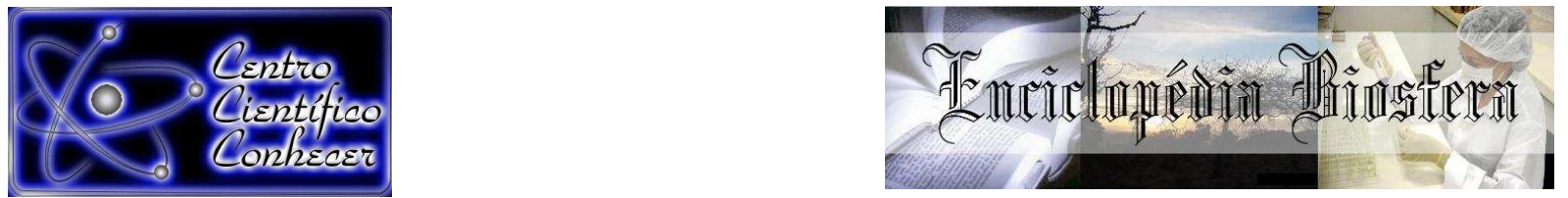

\title{
RELAÇÃO DO POLIMORFISMO DO GENE TP53 NO CÓDON 72 COM CÂNCER DE MAMA: UMA ATUALIZAÇÃO DE METANÁLISE (2002-2015)
}

\footnotetext{
Simone Souza Fagundes ${ }^{1}$, Antonio Márcio Teodoro Silva ${ }^{2}$, Flávia Rodrigues Melo³, Flávio Monteiro Ayres ${ }^{4}$
}

${ }^{1}$ Mestre em Genética pela Pontifícia Universidade Católica de Goiás (PUC GO). biologiasimone@hotmail.com, Goiás-Brasil.

${ }^{2}$ Professor Doutor do Programa em Ciências Ambientais e Saúde da Pontifícia Universidade Católica de Goiás (PUC GO).

${ }^{3}$ Professora Doutora do Programa em Genética da Pontifícia Universidade Católica de Goiás (PUC GO) e do Curso de Ciências Biológicas da Universidade Estadual de Goiás (UEG).

${ }^{4}$ Professor Doutor do Programa de Ciências Moleculares e do Programa de Ciências Aplicadas a Produtos para a Saúde da Universidade Estadual de Goiás (UEG).

Recebido em: 03/10/2016 - Aprovado em: 21/11/2016 - Publicado em: 05/12/2016 DOI: 10.18677/EnciBio 2016B 138

\begin{abstract}
O câncer de mama é o mais incidente na população feminina mundial e brasileira. É uma doença complexa que não tem causa única, resultante da interação de múltiplos fatores. O polimorfismo do gene TP53, no éxon 4 do códon 72 (R72P), inclui um códon codificante do resíduo de aminoácido arginina $(R)$ ou de uma prolina $(P)$ na posição 72 da proteína supressora de tumor p53. Uma vez que os dados relatados mundialmente para o polimorfismo $\mathrm{R} 72 \mathrm{P}$ em câncer de mama são conflitantes e sujeitos à variabilidade de fatores étnicos e geográficos, o presente estudo teve como objetivo atualizar uma metanálise com robusto conjunto de dados sobre o polimorfismo $\mathrm{R} 72 \mathrm{P}$ em pacientes com câncer de mama. A metanálise reuniu 46 artigos somando 26.779 casos e 27.577 controles. Quando analisados os dados globais e por continente, uma associação significativa foi observada entre o risco de desenvolvimento do câncer de mama e o alelo $P$ codificante de prolina.
\end{abstract}

RESUMO

PALAVRAS-CHAVE: Arg72Pro, suscetibilidade genética, p53. 


\title{
TP53 CODON 72 POLYMORPHISM AND BREAST CANCER: A META-ANALYSIS UPDATE (2002-2015)
}

\begin{abstract}
Breast cancer is the most incident among women in Brazil and worldwide. It is a complex disease with several causes, resulting the interactions of multiple factors. The TP53 polymorphism in codon $72(\mathrm{R} 72 \mathrm{P})$, exon 4 , encodes an arginine $(\mathrm{R})$ or a proline $(\mathrm{P})$ in the position 72 of the p53 tumor supressor protein. The present study was conducted considering the controversy background about R72P in breast cancer, likely due to ethnic and geographic factors. In this context, we aimed to update a metaanalysis composed by a large sample size about R72P in breast cancer. The metaanalysis had 46 references included with 26,779 breast cancer cases and 27,577 controls. The proline $(\mathrm{P})$ allele was found associated with the risk of breast cancer development when the meta-analysis was conducted by continente or with global data.

KEYWORDS: Arg72Pro; genetic susceptibility; p53.
\end{abstract}

\section{INTRODUÇÃO}

O câncer de mama é uma das doenças mais comuns em mulheres, resultan ${ }^{\text {tn }}$ da interação entre fatores genéticos e ambientais (ZHUO et al., 2009). A doença ..m prognóstico variado, no qual os fatores genéticos e ambientais exercem peso fundamental para o risco de desenvolvimento e progressão dos casos (ACHATZ et al., 2009). Nesse contexto, o status da proteína p53 é considerado como importante fator prognóstico, tendo a p53 mutada uma forte correlação com uma piora no prognóstico (GIACOMAZZI et al., 2013). No que diz respeito ao polimorfismo do códon 72, dezenas de estudos foram conduzidos nas últimas décadas para analisar a associação entre esse SNP (polimorfismo de nucleotídeo único, do inglês, Single Nucleotide Polymorphism) e o risco de desenvolvimento do câncer de mama em diferentes populações (GIACOMAZZI et al., 2013).

O gene supressor de tumor TP53 se situa no braço curto do cromossomo 17 (17p13.1), com 19.198 nucleotídeos, 11 éxons, dos quais o primeiro não é codificante, e codifica a fosfoproteína nuclear p53, com 393 aminoácidos (WENG et al., 2012). A progressão para o carcinoma se dá com a perda de $17 \mathrm{p}$ e, ao analisá-lo em tumores humanos, identificou-se que o alelo selvagem é frequentemente perdido durante 0 desenvolvimento tumoral, indicando que ambos os alelos são inativados na maioria dos tumores, um alelo por mutação pontual e o outro por deleção (GIACOMAZZI et al., 2013).

Na proteína p53, assim denominada devido ao peso molecular de $53 \mathrm{kDa}$, o polimorfismo do gene TP53, no éxon 4 do códon 72, resulta na substituição de Arginina (Arg) ou Prolina (Pro), que pode codificar um aminoácido arginina (p53Arg) ou uma prolina (p53Pro). Para alguns pesquisadores, a variante Arg é mais eficaz em induzir a célula a apoptose, enquanto que Pro induz a parada do ciclo celular e reparo a danos ao DNA (GIACOMAZZI et al., 2013). O presente estudo teve como objetivo atualizar a metanálise de GONÇALVES et al. (2014), que envolve um amplo grupo de estudo com dados conflitantes sobre o polimorfismo R72P no câncer de mama. 


\section{MATERIAL E MÉTODOS}

A busca de artigos para esta metanálise foi direcionada para artigos publicados sobre o polimorfismo R72P em pacientes com câncer de mama. A busca foi realizada nas bases de dados SciELO, Pubmed, Medline, Cochrane, Capes, Ibecs, Wholis e Lilacs. Foram usadas como palavras-chave "p53", "breast cancer" e "polymorphism". Os critérios para inclusão das referências foram: a) publicação entre os anos 2002 e 2015; b) estudos caso-controle; c) estudos com descrição das frequências genotípicas; d) genotipagem realizada por método de PCR, PCR-RFLP ou sequenciamento de DNA; e) estudos primários; f) disponibilizados na íntegra em bases de dados nacionais ou internacionais; e g) artigos publicados em inglês.

As referências levantadas foram inicialmente selecionadas pelo teste de relevância I, que consiste na análise do resumo ou abstract. No teste de relevância II, os artigos selecionados foram analisados na íntegra para posterior inclusão no estudo e extração dos dados. A análise estatística inerente à metanálise foi feita utilizando-se o programa BioEstat ${ }^{\Theta}$ 5.3. Os dados extraídos das referências foram submetidos ao teste de heterogeneidade do $\chi^{2}$, seguido pelo teste de DerSimonian-Laird (efeito randômico) em caso de homogeneidade ou o teste de Mantel-Haenszel (efeito fixo) em caso de heterogeneidade (SANTOS, 2013). Os resultados foram representados em gráficos Forest plot como resultado das combinações do Odds Ratio e os intervalos de confiança (IC), onde cada estudo é representado por uma linha, sendo que a última adquire o formato de um losango representando a combinação de todos os resultados. Cada estudo possui em uma linha numérica, um gráfico em forma de um quadrado central que é a representação do risco relativo, quanto maior o quadrado maior peso e significância possui o estudo para compor os resultados, e a linha é o intervalo de confiança (IC), e quanto menor o IC e mais próximo estiver da nulidade (posição 1,0 no gráfico), maior importância possui o estudo (PINTO, 2013).

\section{RESULTADOS E DISCUSSÃO}

Um total de 579 referências foi identificado. Destas, 573 foram selecionadas por atenderem inicialmente aos critérios de busca. A aderência da referência ao tema da pesquisa foi avaliada pelo teste de relevância I, após o qual sobraram 304 referências. Conforme ilustrado na Figura 1, 46 referências foram incluídas após o teste de relevância II, cujos dados extraídos somaram 26.779 casos e 27.577 controles.

$\mathrm{Na}$ metanálise, para os modelos dominantes RR+RP vs. PP e RR vs. RP+PP, foram calculados os Odds Ratio, usando o modelo de efeito randômico DerSimonianLaird. Não foi encontrado resultado significativo de risco aumentado para o câncer de mama quando calculado os genótipos associados: RR vs. RP + PP (OR=1,20; 1,071,$34 ; \chi^{2}=335,71$ ) para o alelo $R$ homozigoto dominante (Tabela 1 e Figura 2 ). 


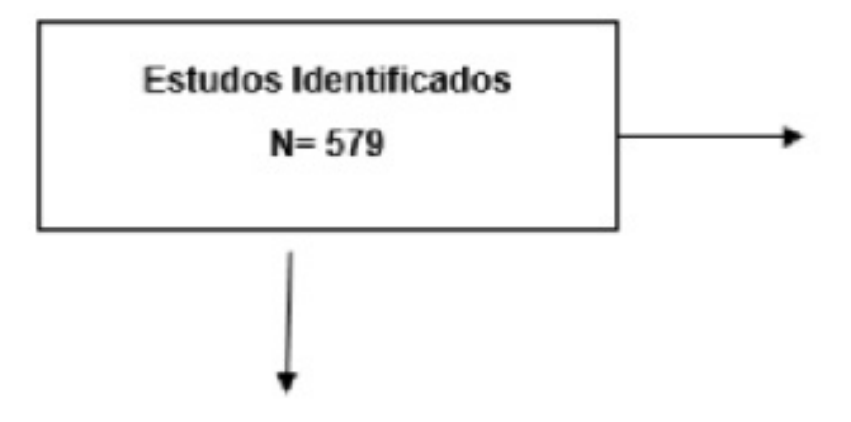

\begin{tabular}{|l|l|}
\hline \multicolumn{2}{|l|}{ Estudos excluidos por repetição, } \\
indisponibilidade na íntegra, \\
idioma diferentes $\quad$ dos \\
estabelecidos para pesquisa $\mathrm{N}=6$
\end{tabular}

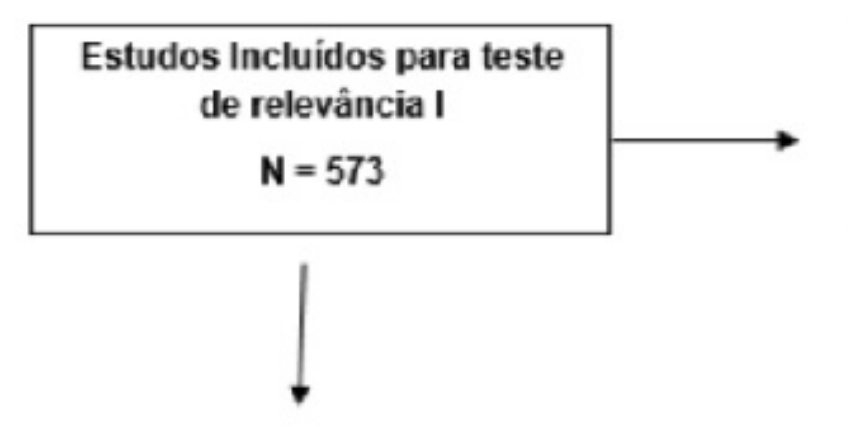

\begin{tabular}{l}
$\begin{array}{c}\text { Estudos excluidos após Teste de } \\
\text { Relevância I } \\
\mathbf{N}=\mathbf{2 6 8}\end{array}$ \\
\hline
\end{tabular}
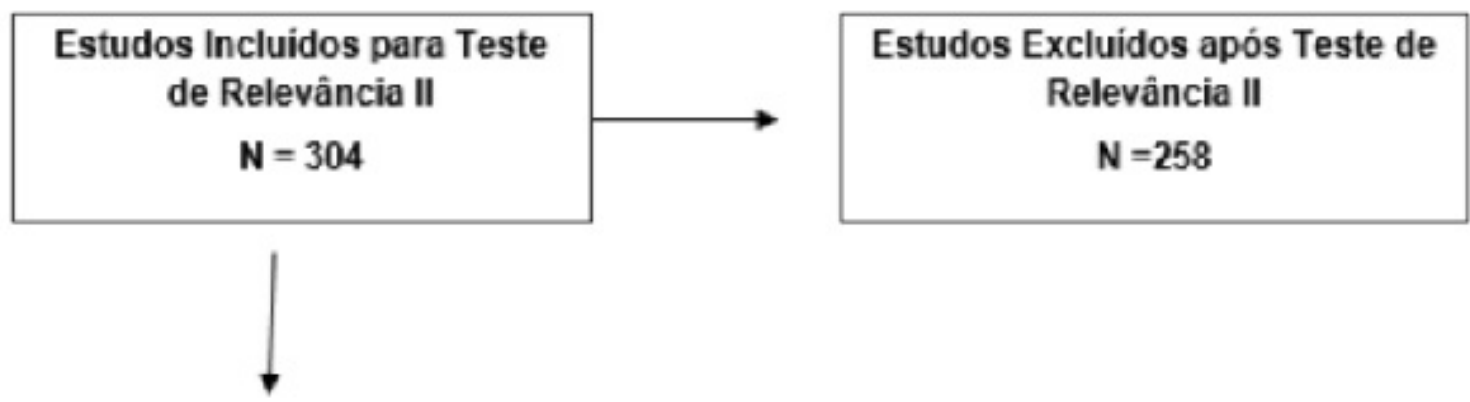

Estudos Incluidos na pesquisa

para realizar metanálise

$\mathbf{N}=\mathbf{4 6}$

FIGURA 1. Esquema da busca feita para a metanálise. Fonte: autor Simone Souza Fagundes. 
TABELA 1. Metanálise do polimorfismo do códon 72 do gene TP53 em câncer de mama.

\begin{tabular}{|c|c|c|c|c|}
\hline Estudo/Continente & $\begin{array}{c}\text { № de casos/ } \\
\text { Controle }\end{array}$ & $\begin{array}{l}\text { RR+RP x PP } \\
\text { OR }(95 \% I C)\end{array}$ & $\begin{array}{c}\text { RRxRP + PP } \\
\text { OR (95\%IC) }\end{array}$ & $\begin{array}{c}\text { R } \times \text { P } \\
\text { OR (95\%IC) }\end{array}$ \\
\hline & $\sum=189 / 181$ & $1.13(0.64-2.01)$ & $1.40(0.91-2.15)$ & $1.16(0.86-1.56)$ \\
\hline Mabrouk et al, 2003 & $30 / 49$ & $0.19(0.65-2.19)$ & $1.21(0.75-1.96)$ & $1.15(0.83-1.61)$ \\
\hline Trifa et al, 2010 & $159 / 132$ & $0.78(0.18-3.40)$ & $2.32(0.93-5.78)$ & $1.59(0.78-3.27)$ \\
\hline América & $\Sigma=7.233 / 8.555$ & $1.60(0.88-1.27)$ & $1.161(0.97-1.40)$ & $1.13(0.98-1.31)$ \\
\hline Damin et al, 2006 & $118 / 202$ & $2.05(0.83-5.09)$ & $2.22(1.40-3.53)$ & $1.79(1.25-2.55)$ \\
\hline Aoki et al, 2009 & $72 / 90$ & $1.78(0.48-6.60)$ & $2.47(1.31-4.66)$ & $1.85(1.14-3.00)$ \\
\hline Gaudet et al, 2008 & $578 / 390$ & $1.11(0.70-1.76)$ & $0.78(0.61-1.01)$ & $0.88(0.71-1.08)$ \\
\hline Cox et al, 2007 & $1.477 / 2.224$ & $0.83(0.63-1.08)$ & $0.92(0.81-1.05)$ & $0.92(0.83-1.02)$ \\
\hline Garcia-Closas et al, 2007 & $2.585 / 3.251$ & $0.92(0.75-1.12)$ & $0.94(0.84-1.04)$ & $0.95(0.87-1.03)$ \\
\hline Sprague et al, 2007 & $1.653 / 1.854$ & $1.16(0.89-1.52)$ & $1.00(0.87-1.14)$ & $1.02(0.92-1.14)$ \\
\hline Lopes et al, 2014 & $50 / 115$ & $1.49(0.43-5.18)$ & $1.043(0.5-2.05)$ & $1.13(0.65-2.0)$ \\
\hline Vymetalkova et al, 2015 & $700 / 611$ & $1.05(0.70-1.56)$ & $1.15(0.93-1.44)$ & $1.10(0.92-1.30)$ \\
\hline Ásia & $\sum=4.051 / 4.169$ & $1.01(0.88-1.16)$ & $1.06(0.91-1.23)$ & $1.04(0.93-1.16)$ \\
\hline Lum et al, 2008 & $393 / 80$ & $0.69(0.37-1.30)$ & $0.64(0.39-1.06)$ & $0.73(0.51-1.03)$ \\
\hline Gochhait et al, 2007 & $243 / 333$ & $1.66(1.12-2.46)$ & $1.85(1.28-2.67)$ & $1.56(1.23-1.92)$ \\
\hline Huang et al, 2003 & $200 / 282$ & $0.54(0.32-0.92)$ & $0.70(0.48-1.02)$ & $0.72(0.55-0.93)$ \\
\hline Ma et al, 2006 & $404 / 472$ & $1.14(0.82-1.59)$ & $1.25(0.95-1.66)$ & $1.16(0.96-1.40)$ \\
\hline Rajkumar et al, 2008 & $250 / 500$ & $1.27(0.89-1.80)$ & $0.97(0.69-1.37)$ & $1.08(0.87-1.34)$ \\
\hline Li et al, 2002 & $28 / 50$ & $1.38(0.48-3.99)$ & $2.54(0.93-6.94)$ & $1.68(0.87-3.27)$ \\
\hline Alawadi et al, 2011 & $288 / 188$ & $6.12(2.66-14.08)$ & $1.08(0.71-1.63)$ & $1.31(1.00-1.71)$ \\
\hline Suresh et al, 2011 & $35 / 37$ & $2.28(0.59-8.91)$ & $0.95(0.35-2.57)$ & $1.21(0.62-2.34)$ \\
\hline Kazemi et al, 2009 & $42 / 57$ & $0.05(0.003-0.89)$ & $0.65(0.23-1.84)$ & $0.65(0.37-1.15)$ \\
\hline Singh et al, 2008 & $104 / 105$ & $0.91(0.40-2.06)$ & $2.26(1.21-3.85)$ & $1.42(0.96-2.11)$ \\
\hline Khadang et al, 2007 & $221 / 205$ & $1.60(0.95-2.68)$ & $1.04(0.70-1.54)$ & $1.17(0.89-1.54)$ \\
\hline Siddique et al, 2005 & $94 / 265$ & $0.62(0.34-1.12)$ & $0.92(0.57-1.49)$ & $0.83(0.59-1.16)$ \\
\hline Noma et al, 2004 & $191 / 218$ & $0.93(0.54-1.60)$ & $0.92(0.62-1.35)$ & $0.82(0.61-1.10)$ \\
\hline Katiyar et al, 2003 & $77 / 41$ & $2.79(0.93-8.39)$ & $1.22(0.51-2.95)$ & $1.38(0.80-2.36)$ \\
\hline Saadatian et al, 2014 & $100 / 100$ & $0.74(0.4-1.38)$ & $1.86(0.88-3.9)$ & $1.062(0.71-1.57)$ \\
\hline Sharma et al, 2014 & $200 / 200$ & $0.8(0.50-1.3)$ & $0.61(0.4-0.95)$ & $0.755(0.57-0.99)$ \\
\hline Gohari-Lasaki et al, 2015 & $100 / 100$ & $0.53(0.25-1.2)$ & $1.0(0.55-1.81)$ & $0.83(0.56-1.23)$ \\
\hline Suspitsin et al., 2003 & $529 / 393$ & $0.86(0.52-1.41)$ & $1.04(0.80-1.35)$ & $1.00(0.81-1.23)$ \\
\hline Wang-Gohrke et al., 2002 & $552 / 543$ & $0.82(0.53-1.26)$ & $0.85(0.67-1.07)$ & $0.87(0.72-1.05$ \\
\hline Europa & $\Sigma=15.306 / 13.672$ & $1.03(0.95-11.25)$ & $1.13(1.00-1.27)$ & $1.02(0.99-1.06)$ \\
\hline Sinilnikova et al, 2009 & $3.959 / 3.052$ & $0.97(0.80-1.16)$ & $1.01(0.92-1.11)$ & $1.00(0.93-1.08)$ \\
\hline Cavallone et al, 2008 & $157 / 112$ & $1.29(0.52-3.21)$ & $1.00(0.62-1.63)$ & $1.04(0.71-1.53)$ \\
\hline Baynes et al, 2007 & $2.023 / 2.197$ & $1.04(0.82-1.30)$ & $1.05(0.93-1.18)$ & $1.04(0.95-1.14)$ \\
\hline Tommiska et al, 2005 & $1.551 / 733$ & $1.02(0.72-1.43)$ & $0.93(0.78-1.11)$ & $0.96(0.83-1.10)$ \\
\hline
\end{tabular}




\begin{tabular}{|c|c|c|c|c|}
\hline Akkiprik et al, 2009 & $95 / 108$ & $039(0.14-1.12)$ & $1.38(0.78-2.42)$ & $1.02(0.65-1.91)$ \\
\hline Kara et al, 2010 & $204 / 192$ & $1.48(0.73-3.00)$ & $1.45(0.98-2.15)$ & $1.34(0.99-1.81)$ \\
\hline Bisof et al, 2010 & $95 / 107$ & $0.48(0.22-1.04)$ & $0.48(0.27-0.87)$ & $0.58(0.39-0.86)$ \\
\hline Denisov et al, 2009 & $297 / 275$ & $1.28(0.73-2.23)$ & $0.87(0.62-1.20)$ & $0.96(0.75-1.25)$ \\
\hline Hernandez et al, 2009 & $135 / 295$ & $1.60(0.72-3.54)$ & $0.90(0.60-1.36)$ & $1.03(0.74-1.43)$ \\
\hline Costa et al, 2008 & $248 / 646$ & $0.81(0.49-1.32)$ & $0.86(0.64-1.16)$ & $0.87(0.69-1.10)$ \\
\hline Buyru et al, 2007 & $115 / 63$ & $1.44(0.58-3.57)$ & $1.77(0.96-3.29)$ & $1.52(0.96-2.43)$ \\
\hline Johnson et al, 2007 & $472 / 2.462$ & $1.17(0.79-1.74)$ & $0.98(0.80-1.19)$ & $1.01(0.87-1.19)$ \\
\hline Schimidt et al, 2007 & $5.191 / 3.834$ & $1.01(0.86-1.18)$ & $1.05(0.96-1.14)$ & $1.03(0.96-1.10)$ \\
\hline Kalemi et al, 2005 & $42 / 51$ & $2.52(0.69-9.26)$ & $6.35(2.54-15.84)$ & $3.29(1.73-6.25)$ \\
\hline Ohayon et al, 2005 & $132 / 167$ & $4.86(1.52-15.53)$ & $4.29(4.68-6.96)$ & $3.10(2.10-4.56)$ \\
\hline Menzel et al, 2004 & $475 / 302$ & $1.64(0.97-2.76)$ & $1.25(0.94-1.67)$ & $1.27(1.01-1.60)$ \\
\hline Bayru et al, 2003 & $115 / 76$ & $1.61(0.69-3.73)$ & $3.23(1.7-6.00)$ & $2.09(1.36-3.22)$ \\
\hline Total & $\begin{array}{c}26,779 / 27,577=5 \\
4,356\end{array}$ & $\begin{array}{c}1.06(0.95-1.18) \\
\chi^{2}=88.66 \\
\mathrm{p}=0.0001\end{array}$ & $\begin{array}{c}1.20(1.07-1.34) \\
\chi^{2}=335.71 \\
p=0.0001\end{array}$ & $\begin{array}{c}1.16(1.05-1.28) \\
\chi^{2}=444.31 \\
p=0.0001\end{array}$ \\
\hline
\end{tabular}

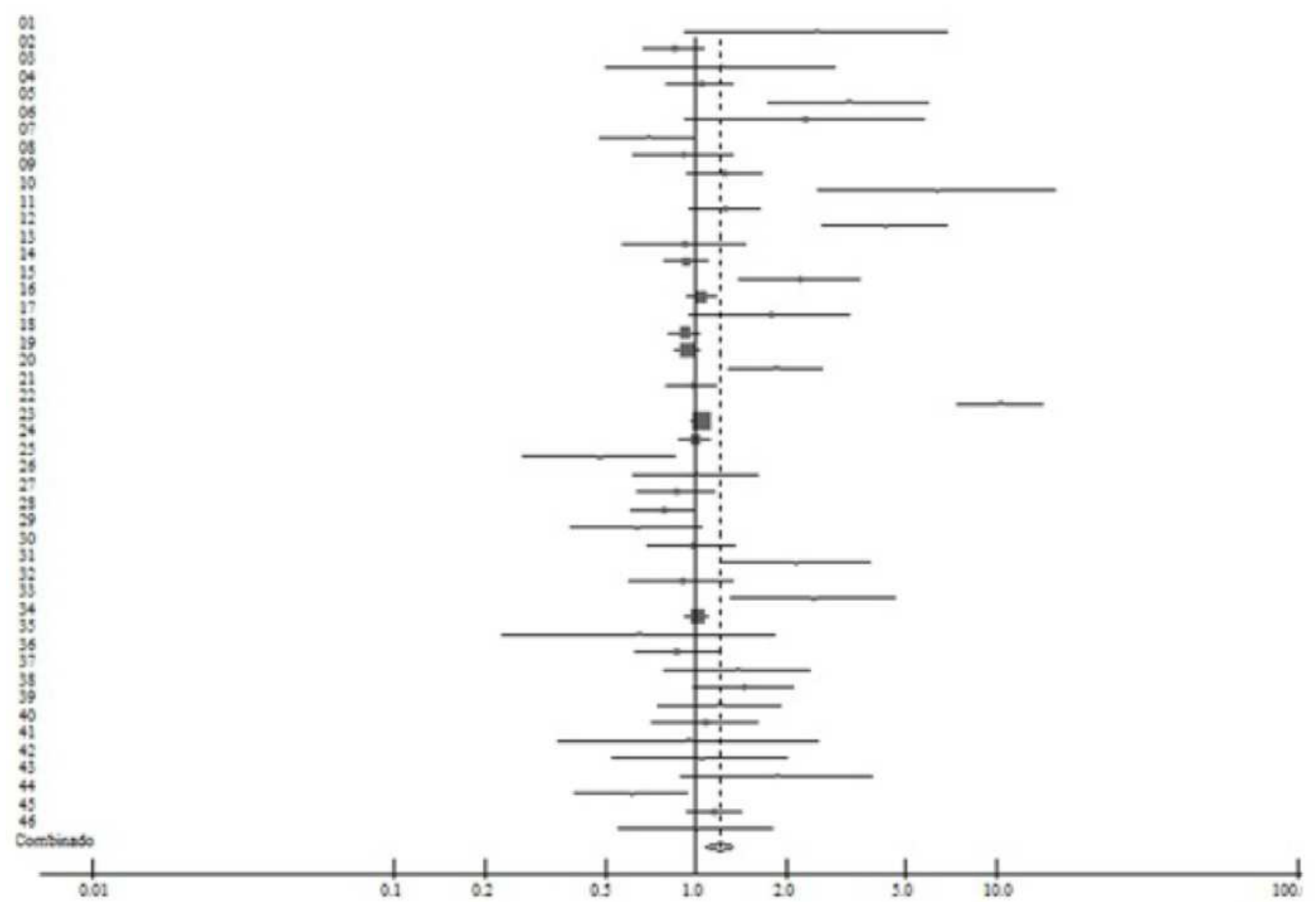

FIGURA 2. Metanálise do modelo dominante RR $x$ RP + PP. Fonte: Autor: Simone Souza Fagundes. 
Ao analisar os resultados de associação do genótipo dominante RR $\times$ RP + PP $(1,06$; $\left.0,95-1,18 ; \chi^{2}=88,66\right)$, percebeu-se diferença significativa para 0 alelo $P$ em relação ao desenvolvimento da tumorigênese (Figura 3 ).

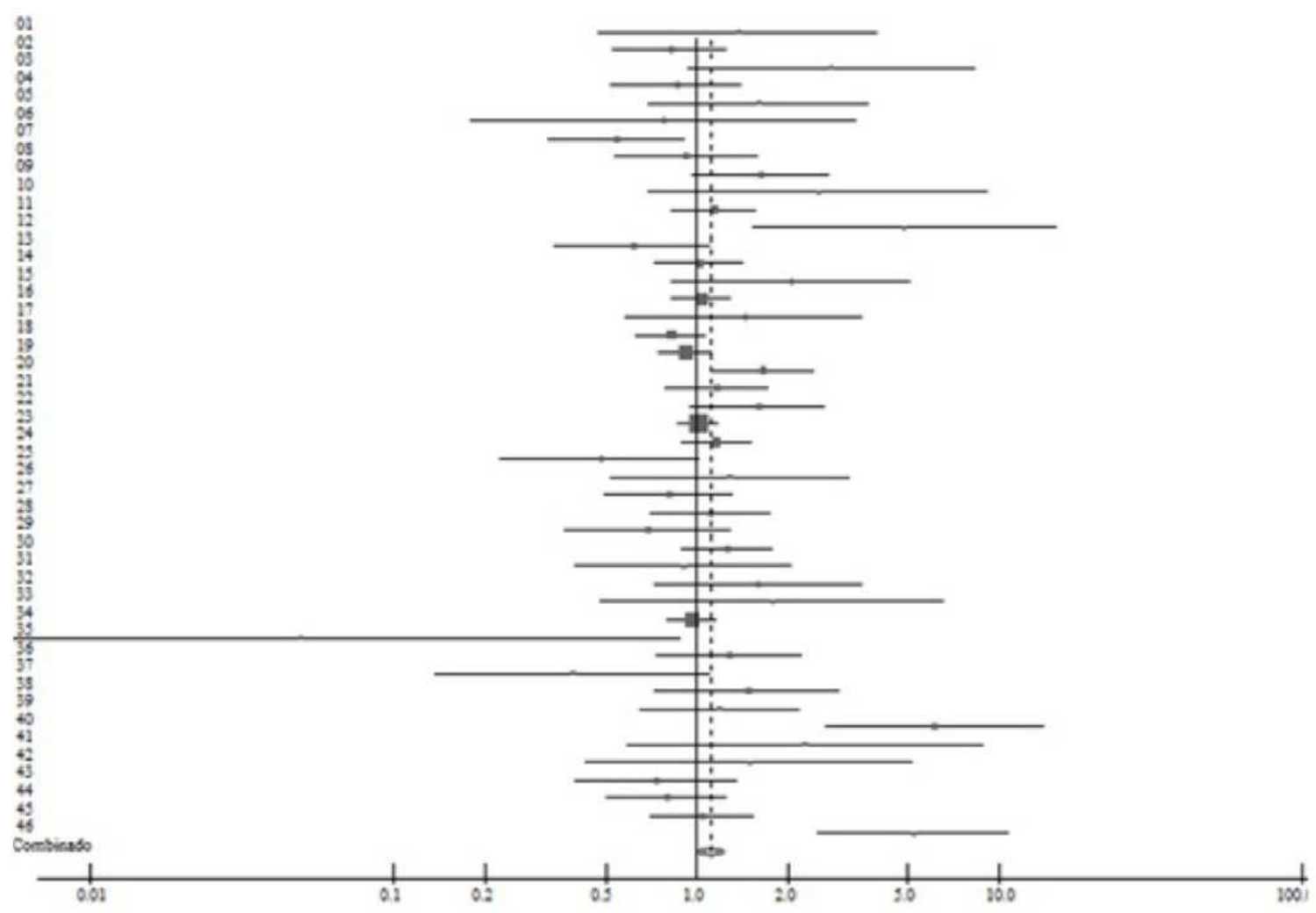

FIGURA 3. Metanálise do modelo dominante RR+RP x PP.

Fonte: Autor: Simone Souza Fagundes.

Um dos fatores relacionados a resultados tão divergentes está associado ao câncer de mama apresentar variação étnica e geográfica muito grande (AI-QUASEN et al., 2012). Indivíduos que saem de regiões de baixa incidência de câncer de mama e imigram para países com alta taxa de incidência, como é o caso dos Estados Unidos, mostram que os casos da doença aumentam progressivamente em gerações. Já o Brasil, por ser um país bastante heterogêneo em todos os aspectos, devido à grande extensão territorial e diversidade étnica e cultural, as taxas de câncer de mama também são diferentes para cada região (Al-QUASEN et al., 2012). Por outro lado, estudos de SHARMA et al. (2014), SAADATIAN et al. (2014) e GOHARI-LASAKI et al. (2015) apresentaram resultados contrários, mostrando que o genótipo heterozigoto RP foi mais frequente, porém sem valor significativo importante. Assim como os resultados encontrados nesta metanálise, LOPES et al. (2014) e VYLMETALKOVA et al. (2015), relataram dados que demonstraram a prevalência do homozigoto $\mathrm{R}$ como mais impactante na interferência com o câncer de mama. 
O desenvolvimento da tumorigênese pelo gene TP53 representa risco aumentado do genótipo $R$ homozigoto (62\%) em comparação com o genótipo $P$ homozigoto (21\%) (Al-QUASEN et al., 2012). O polimorfismo do alelo R no códon 72 é um potencial fator de risco para o câncer de mama, enquanto que o RP heterozigoto é considerado um fator de proteção contra o câncer de mama (DAMIN et al., 2006). Tais autores descobriram que o genótipo RR foi significativamente associado com um risco aumentado para câncer de mama $(O R=2,9 ; \mathrm{IC} 95 \%=1,43-3,6 ; p<0,002)$. As frequências alélicas de p53R e p53P variam etnogeograficamente sugerindo que os dois alelos podem sofrer pressão seletiva nas diferentes populações étnicas, o que pode ser devido a uma adaptação ecológica (SIDDIQUE et al). Fatores étnicos e geográficos, além de variação da frequência alélica em diferentes populações, podem explicar o papel controverso do polimorfismo R72P com câncer de mama (GONÇALVES et al., 2014).

Ao analisar as frequências obtidas por continentes, observou-se que no continente Africano e Asiático houve frequência mais elevada do genótipo heterozigoto $\mathrm{RP}$ em relação as variantes homozigotas, mostrando, porém, pouca relevância para o desenvolvimento da carcinogênese (SHARMA et al., 2014). Em concordância com as atualizações feitas, a metanálise de GONÇALVES et al. (2014) também obteve maior proporção genotípica entre heterozigotos na Ásia e na África. Nos continentes Americano e Europeu, observou-se incidência aumentada do polimorfismo do códon 72 para o alelo prolina homozigoto, que está de acordo com os resultados de GONÇALVES et al. (2014).

É provável que o alelo $\mathrm{P}$ seja o mais antigo, sua frequência é de aproximadamente 60 a $70 \%$ em afrodescendentes, mas de apenas 10 a $35 \%$ em caucasianos (LEVINE, et al., 2006). A frequência do alelo $P$ aumenta de maneira linear à medida que as populações se aproximam da linha equatorial o que sugere a hipótese de que a alta exposição a luz UV resultou na seleção do alelo $P$, sugerindo que este seja um alelo protetor mais eficiente contra doenças induzidas pela exposição à luz solar (DUMONT et al., 2003; LEVINE et al., 2006; MURPHY, 2006).

A frequência do alelo $R$ no códon 72 da população caucasiana, em geral, é de aproximadamente $70 \%$ (DONEHOWER et al., 2005). Pesquisas já foram conduzidas com o intuito de relacionar os diversos polimorfismos do gene TP53 como marcador para compreender a distribuição populacional na América Latina (MOURA-GALLO et al., 2005).

\section{CONCLUSÕES}

Em conclusão, encontrou-se associação significativa entre o polimorfismo do códon 72 no gene TP53 e o câncer de mama. Os dados globais mostraram um risco aumentado para o modelo dominante do alelo e do genótipo $P$ homozigoto dominante apenas na Europa, nos outros continentes não foram encontrados resultados significantes. A presente metanálise se apoia em uma grande amostra composta por 26.779 casos e 27.577 controles, somando 54.356 indivíduos extraídos de 46 referências de difusão internacional. 


\section{AGRADECIMENTOS}

Esse estudo foi fomentado pela Fundação de Amparo à Pesquisa do Estado de Goiás (FAPEG), No. 201201268940872). SSF e FMA foram contemplados com bolsas da FAPEG (chamada 3/2014) e da Universidade Estadual de Goiás, UEG (Programa de Incentivo à Pesquisa e Produção Científica, PROBIP), respectivamente. As discussões com os professores Andréia Juliana Rodrigues Caldeira (UEG) e Cláudio Carlos da Silva (PUC-Goiás e UEG) contribuíram valiosamente para esta pesquisa.

\section{REFERÊNCIAS}

ACHATZ, M,I; HAINAUT, P; ASHTON-PROLLA, P. Highly prevalent TP53 mutation predisposing to many cancers in the Brazilian population: a case for newborn screening? Lancet Oncol. v. 10, n. 9, p. 920-5, 2009. Disponível em $<$ http://www.thelancet.com/pdfs/journals/lanonc/PIIS1470-2045(09)70089-0.pdf> doi: doi.org:// dx 10.1016/S1470-2045(09)70089-0.

AI-QUASEM, A; TOULIMAT, M; TULBAH, A; ELKUM, N; AL-TWEITERI, T; ABOUSSEKHRA, A. The p53 codon 72 polymorphism is associated with risk and early onset of breast cancer among Saudi women. Oncol Lett. v. 3, p. 875-8, 2012. doi.org:// dx. 10.3892/ol.2012.581.

DAMIN AP; FRAZZON AP; DAMIN DC; ROEHE A; HERMES V; ZETTLER C; ALEXANDRE CO. Evidence for an association of TP53 codon 72 polymorphism with breast cancer risk. Cancer Detect Prev. 2006; 30: 523 529.:doi:http://dx.doi.org/10.1016/j.cdp.2006.09.007.

DUMONT P; LEU JI; DELLA PIETRA III AC; GEORGE DL; MURPHY M. The codon 72 polymorphic variants of p53 have markedly different apoptotic potencial. Nature Genetics; 33: 357-365. 2003. http: doi.org://dx 10.1038/ng1093.

GIACOMAZZI J; SELISTRE S; DUARTE J; RIBEIRO JP; VIEIRA P J; DE SOUZA MACEDO ROSSI C; CZEPIELEWSKI M; NETTO CB; HAINAUT P. TP53 pR337H is conditional cancer-predisposing mutation: further evidence from homozygous patient. BMC Cancer, 13, 187. 2013. http: doi.org://dx 10.1186/1471-2407-13-187.

GOHARI-LASAKI S; GHARESOURAN J; GHOJAZADEH M; MONTAZERI V; ARDEBI SMM. Lack of Influence of TP53 Arg72Pro and 16bp Duplication Polymorphisms on Risk of Breast Cancer in Iran. Asian Pacific Journal of Cancer Prevention, Vol 16, 2015. http: doi.org: //dx 10.4103/0019-509X.84925.

GONÇALVES ML; SARAH MB; CORDEIRO JABL; Saddi V-A; AYRES FM; VILANOVACOSTA AST; SILVA AMTC Association of the TP53 codon 72 polymorphism and breast cancer risk: a meta-analysis. Springer Plus, 2014. http://dx: doi.org: 10.1186/21931801-3-749. 
LEVINE AJ; HU W; FENG Z. The P53 pathway: what questions remain to be explored? Cell Death Differ; 13: 1027-1036. 2006. http://dx:doi.org:10.1038/sj.cdd.4401910.

LOPES LF; GUEMBAROVSKI RL; GUEMBAROVSKI AL; KISHIMA MO; CAMPOS CZ; DEROSSI DR; ARIZA CB; MUROBUSHI OZAWA PM; OLIVEIRA CEC; BANIN-HIRATA BK; VITIELLO GAF; BORELLI SD; WATANABE MAE. Protein Expression and Codon 72 Polymorphism of TP53 Gene in Triple Negative Breast Cancer . Braz. Arch. Biol. Technol. v.57 n.6: pp. 895-899, Nov/Dec 2014. http://dx.doi.org/10.1590/S15168913201402559 ISSN 1516-8913

MOURA-GALLO CV. Mutação no gene TP53 em tumores malignos de mama: associação com fatores de risco e características clínico-patológicas, inclusive risco de óbito, em pacientes residentes no Rio de Janeiro. Revista Brasileira de Epidemiologia, v.7 n2 p.167-175, 2005. http://dx.doi.org/10.1590/S1415$790 \times 2004000200006$.

MURPHY ME. Polymorphic variants in the p53 pathway. Cell Death Differ ;13, 916-20. 2006 http://dx doi.org:10.1038/sj.cdd.4401907

PINTO CM. "Metanálise qualitativa como abordagem metodológica para pesquisas em letras". Atos de Pesquisa em Educação. v. 8, n. 3, p. 1033 - 1048, set. dez. 2013 doi http://dx.doi.org/10.7867/1809-0354.2013 v8 n3 p1033-1048.

SAADATIAN Z; GHARESOURAN J; GHOJAZADEH M; GHOHARI-LASAK; S;TARKESH-ESFAHANI N; MOHADDES SM. Association of rs1219648 in FGFR2 and rs1042522 in TP53 with Premenopausal Breast Cancer in an Iranian Azeri Population. Asian Pac J Cancer Prev, 15 (18), 7955-7958 2014. doi: http://dx.doi.org/10.7314/apjcp.2014.15.18

SANTOS EJF; CUNHA M. Interpretação crítica dos resultados estatísticos de uma metaanálise: estratégias metodológicas. Millenium 2013, 44:85-98. http://dx.doi.org/10.12707/RIV15055

SHARMA S; SAMBYAL V; GULERIA K; MANJARI M; SUDAN M; UPPAL MS; SINGH NR; BANSAL D; GUPTA A. TP53 Polymorphisms in Sporadic North Indian Breast Cancer Patients. Asian Pacific Journal of Cancer Prevention, Vol 15, 2014. doi:http://dx.doi.org/10.7314/APJCP.2014.15.16.6871

SIDDIQUE MM; BALRAM C; FISZER-MALISZEWSKA L; AGGARWAL A; TAN A; TAN P; SOO KC; SABAPATHY K (2005) Evidence for selective expression of the p53 codon 72 polymorphs: implications in cancer development. Cancer Epidemiol http:// dx.doi.org: 10.1186/2193-1801-3-749

VYMETALKOVA V; SOUCEK P; KUNICKA T; JIRASKOVA K; BRYNYCHOVA V; PARDINI B. Genotype and Haplotype Analyse of TP53 Gene in Breadt Cancer Patients: 
Association with Risk Clinica, Outcomos. Plosone |http://dx.doi.org:10.1371/journal.pone.0134463 July30,2015.

WENG Y; LU L; YUAN G; GUO J; ZHANG Z; XIE X; CHEN G; ZHANG J. p53 codon 72 polymorphism and Hematological Cancer Risk: An Update Meta-Analysis, Plosone, v. 7, n. 9, e-45820, 2012. http:// dx.doi.org:10.1371/journal.pone.0045820

ZHUO W; ZHANG Y; XIANG Z; CAI L CHEN Z. Polymorphisms of TP53 codon 72 with breast carcinoma risk: evidence from 12226 cases and 10782 controls. J Exp Clin

Cancer Res 2009; 28:115. http:// dx. doi.org: 10.1186/1756-9966-28-115 